\title{
Quantitative Analysis of Total Petroleum Hydrocarbons in Soils: Comparison between Reflectance Spectroscopy and Solvent Extraction by 3 Certified Laboratories
}

\author{
Guy Schwartz, ${ }^{1,2,3}$ Eyal Ben-Dor, ${ }^{2}$ and Gil Eshel ${ }^{4}$ \\ ${ }^{1}$ Porter School of Environmental Studies, Tel-Aviv University, Tel-Aviv 69978, Israel \\ ${ }^{2}$ Remote Sensing Laboratory, Tel-Aviv University, Tel-Aviv 69978, Israel \\ ${ }^{3}$ Geography and Human Environment Department, Tel-Aviv University, P.O. Box 39040, Tel-Aviv 69978, Israel \\ ${ }^{4}$ The Soil Erosion Research Station, Ruppin Institute, Emeck Hefer 40250, Israel
}

Correspondence should be addressed to Guy Schwartz, guy.rslab@gmail.com

Received 9 January 2012; Revised 29 March 2012; Accepted 3 April 2012

Academic Editor: Jose Alexandre Melo Dematte

Copyright (๑) 2012 Guy Schwartz et al. This is an open access article distributed under the Creative Commons Attribution License, which permits unrestricted use, distribution, and reproduction in any medium, provided the original work is properly cited.

\begin{abstract}
The commonly used analytic method for assessing total petroleum hydrocarbons (TPH) in soil, EPA method 418.1, is usually based on extraction with 1,1,2-trichlorotrifluoroethane (Freon 113) and FTIR spectroscopy of the extracted solvent. This method is widely used for initial site investigation, due to the relative low price per sample. It is known that the extraction efficiency varies depending on the extracting solvent and other sample properties. This study's main goal was to evaluate reflectance spectroscopy as a tool for TPH assessment, as compared with three commercial certified laboratories using traditional methods. Large variations were found between the results of the three commercial laboratories, both internally (average deviation up to $20 \%$ ), and between laboratories (average deviation up to 103\%). Reflectance spectroscopy method was found be as good as the commercial laboratories in terms of accuracy and could be a viable field-screening tool that is rapid, environmental friendly, and cost effective.
\end{abstract}

\section{Introduction}

Among the chemicals that are relevant as environmental contaminants, petroleum hydrocarbons (PHC) are of particular significance. The widespread use of PHC for transportation, heating and industry has led to the release of these petroleum products into the environment through accidental spills, long-term leakage, or operational failures. Consequently, many soil and water areas are contaminated with PHC. PHC are well known to be neurotoxic to humans and animals. Several studies have been conducted in order to verify the effects of PHC on humans and animals [1-3]. For both the diagnosis of suspected areas and the possibility of controlling the rehabilitation process, there is a great need to measure correctly the amounts of PHC in soils.

Total petroleum hydrocarbons (TPH) is a commonly used gross parameter for quantifying environmental contamination originated by various PHC products such as fuels, oils, lubricants, waxes, and others [4]. Traditional wet chemistry methods for determining TPH level in soil samples is based on extracting the contaminant from the soil sample. The TPH level in the extracted solution is then determined by a gravimetric, FTIR, or GC measurement calibrated by an EPA calibration standard.

The TPH gross parameter is in use worldwide and facilitates an important stage of contaminated sites investigation; therefore, it is important to examine the effects of hydrocarbon type and soil properties on the extraction efficiency, as well as cross-lab repeatability.

The common method for assessing TPH in soil samples is based on a modified version of EPA method 418.1. This method is based on extraction with 1,1,2-Trichlorotrifluoroethane (Freon 113, GC 99.9\%), although other extracting solvents are available (i.e., Carbon tetrachloride, $\mathrm{N}-\mathrm{Hexane}$, etc.). This method was originally introduced in 1978 [5] by the USEPA in order to assess TPH in waste water but was 
TABLE 1: Major soil properties.

\begin{tabular}{lccccccccccc}
\hline $\begin{array}{l}\text { Israeli local } \\
\text { name }\end{array}$ & USDA classification & $\begin{array}{c}\mathrm{HM} \\
\%\end{array}$ & Sand & $\begin{array}{c}\text { Silt } \\
\text { volume } \%\end{array}$ & Clay & SOC & $\begin{array}{c}\text { SIC } \\
\mathrm{g} \mathrm{kg}^{-1}\end{array}$ & $\begin{array}{c}\text { Total N } \\
\mathrm{pH}^{1}\end{array}$ & $\begin{array}{c}\text { EC } \\
\mathrm{mS} \mathrm{m}^{-1}\end{array}$ & $\begin{array}{c}\text { SSA } \\
\mathrm{m}^{2} \mathrm{~g}^{-1}\end{array}$ \\
\hline Loess & Typic xerofluvent & 4.14 & 38.6 & 49.4 & 12 & 5.4 & 22.5 & 0.9 & 8.22 & 5.44 & 167 \\
Hamra & Typic xerocherept & 1.44 & 97.37 & 1.73 & 0.9 & 1.5 & 2.1 & 0.5 & 8.57 & 0.08 & 83 \\
Gromosol & Typic chromoxerert & 5.23 & 46.46 & 38.98 & 14.56 & 7.6 & 12.5 & 1.3 & 8.68 & 0.55 & 238 \\
\hline
\end{tabular}

${ }^{1} 1$ to 2 ratio.

later adjusted in 1983 [6] for the assessment of TPH in soil samples. Newer methods are available for determining TPH in soil samples; these methods are based on extraction with other solvents and are usually followed by gas chromatograph analysis for THP determination. As these methods are more expensive, the EPA method 418.1 is in vast use as a screening tool $[4,7]$.

There are number of possible interactions between inorganic and organic soil components and organic pollutants, soil organic matter, and clays, having significant impact on solid-liquid extraction. Furthermore, the solvent extraction of compounds from soil or sludge samples is dependent on the moisture content in the soil [8]. There are some inherent problems with IR readings of the extracted solvent; all petroleum hydrocarbons do not respond equally to infrared analysis, and comparison of the unknown to a standard mixture may give results with high systematic errors [9]. The major problem with the adjusted EPA 418.1 method is that the extraction yields can be strongly matrix dependent, and the extraction method development and optimization may be quite complicated. These extraction-related problems mainly originate from the diversity of chemical and physical properties of petroleum hydrocarbons, which affect not only the solubility of hydrocarbons to the solvents, but also on the strength of analyte-soil matrix interactions, and therefore render the control of the extraction process of petroleum hydrocarbons from soil problematic.

In conclusion, it is clear that the adjusted EPA method 418.1 may overestimate TPH as a result of the following: (1) differences in infrared molar absorptivity for calibration standards and petroleum products; (2) detection of naturally occurring hydrocarbons; (3) infrared dispersion by mineral particles. Negative bias may also be introduced via (1) poor extraction efficiency of Freon-113 for high-molecularweight hydrocarbons; (2) differences in molar absorptivity; (3) removal of five to six-ring alkylated aromatics during the silica gel cleanup procedure [10].

Quality assurance in the area of TPH determination is under developed and actually, except in few cases [11-13], there have not been any attempts to estimate the uncertainty related to the analytical procedure of TPH determination.

Taking in consideration all the possible biases that can occur during the adjusted EPA 418.1 method, as well as the fact that each laboratory uses somewhat different protocols and equipment for the extraction process and TPH determination; a methodic cross-laboratory evaluation is needed.

In addition to the traditional analytical chemistry methods used for measuring TPH in the soil samples, a new novel method based on reflectance spectroscopy was applied.
Reflectance spectroscopy is commonly applied for quantitative analysis in many disciplines. This method consists of measuring the reflected electromagnetic energy from the soil samples in the VIS-NIR-SWIR region (350-2500 nm), and modeling this spectral data against samples with known concentration levels. Extracting the information about the soil attributes that is hidden within the spectral information, is done by using multivariate statistical techniques, also called chemometrics. Essentially, this involves regression techniques coupled with spectral preprocessing. A more detailed description of the spectral preprocessing and the chemometrics process as well as an overview of reflectance spectroscopy as a tool for monitoring contaminated soils can be found in a recent publication by the authors [7].

The spectral properties of hydrocarbons were identified at the late 1980s, although it was argued that these properties are visible at concentrations of $4 \% \mathrm{wt}$ and above [14]. Several studies were conducted during the past 20 years in the field of PHC and reflectance spectroscopy (ie., [15-24]) that showed the potential of reflectance spectroscopy as being used as a tool for predicting TPH content. For taking a step forward in acceptance of this tool by the environmental protection authorities, a validation study that includes a comparison of the results of commercial laboratories analysis and reflectance spectroscopy performance is needed. Therefore, The goals of this study are (1) a comparison of the inner and interlaboratory TPH measuring capabilities, (2) general accuracy of the measured TPH levels as compared to the known TPH levels of the contaminated soil samples, and (3) Testing reflectance spectroscopy as a viable replacement for the traditional methods based on solvent extraction.

\section{Materials and Methods}

Three certified laboratories in Israel were selected for this study. Analogue soils typical to Israel were artificially contaminated with PHC and sent at the same time and in the same conditions to all laboratories. In addition, the samples underwent a new NIRS procedure that we developed in TAU in which reflectance spectroscopy is used to determine $\mathrm{TPH}$ level [7].

2.1. Soils and Hydrocarbons. Three soils were selected for this study (defined according to Israeli naming system [25] as well as the USDA key to soil taxonomy [26]): Loess (Typic Xerofluvent), Hamra (Typic Xerocherept), and Gromosol (Typic Chromoxerert). These soils represent a wide range of soil properties as described in Table 1 and are significantly differ from each other. The soils were collected from areas that were assumed to have no PHC contamination and 
were air-dried and sieved through a $2 \mathrm{~mm}$ sieve twice. The soils properties were determined by the traditional methods in soil science as follows: hydroscopic moisture content was determined by weight loss after $24 \mathrm{~h}$ at $105^{\circ} \mathrm{C}$. pH level and electrical conductivity were determined with a laboratory bench top $86505 \mathrm{pH} /$ Conductivity meter by M.R.C Ltd. in a $1: 2$ soil and DI water suspension (resp.) after reaching equilibrium (30 minutes). Specific surface area (SSA) was determined by the absorption of mono layer of ethylene glycol monoethyl ether (EGME) [27]. Particle size distributions were determined by Marvin Mastersizer 2000 following Eshel et al. methodology [28]. SOC, SIC, and Total N were determined by a flash CHN elemental analyzer (Thermo Scientific Flash 2000). The soils analogue contaminated samples were prepared by mixing a known weight of several PHC types including: octane fuel, diesel and kerosene with known quantities of soil. For making well-mixed low concentration samples, we initially mixed a batch of $98.5 \mathrm{gr}$ of soil with $1.5 \mathrm{gr}$ of the selected PHC; after mixing the initial batch, the batch was then mixed again with clean soil at three concentration levels. In order to minimize the loss of PHC components, we minimized exposure to open air as much as possible. Each sample was divided equally into 4 amber glass vials, capped with a PTFE lined cap, and kept at $4^{\circ} \mathrm{C}$. Three of the vials were sent to the analytical laboratories for analysis, 1 vial was kept for reflectance spectroscopy analysis. Table 2 describes the samples contamination properties and presents the calculated concentration info.

2.2. Extraction and TPH Measurement Method. The general methodology for the adjusted EPA 418.1 method is based on taking a representative soil sample (3-10 gr.), adding sodium sulfate ( $1-5$ gr.) to absorb any water and adding an extracting solvent (usually Freon 113, 20-30 mL) to the mixture. This mixture is then kept in a sealed glass vial capped with a PTFE cap and placed in a sonic bath for assisting and hasting the extraction process (about 10-45 minutes). Silica gel is then added to the mixture to absorb any polar hydrocarbons (nonfuel-related soil organic matter and fatty acids), and the mixture is mixed well. The filtered extract is then measured in an FTIR spectrometer at $3.42 \mu \mathrm{m}$ (some laboratories use other absorption peaks in the close region). A calibration curve is created by using the 418.1 EPA standard (consists of $31.5 \%$ isooctane, $35 \%$ hexadecane and $33.5 \%$ chlorobenzene) diluted in the same extracting solution at at least 3 concentrations. The absorption depth of the measured sample is then converted to TPH values by the calibration curve. As this method is an adjusted EPA method, it can vary slightly between analytical laboratories, depending on internal laboratory standards, procedures, and equipment. The three laboratories used for analyzing the samples prepared for this study are commercial laboratories, certified by the national laboratories certification authority, thus the exact procedure is confidential and not known to the authors, although the principal remains the same. All 30 contaminated samples prepared for this study as described above were sent to the three certified laboratories for chemical analysis determination of TPH levels, the results are summarized in Table 2.
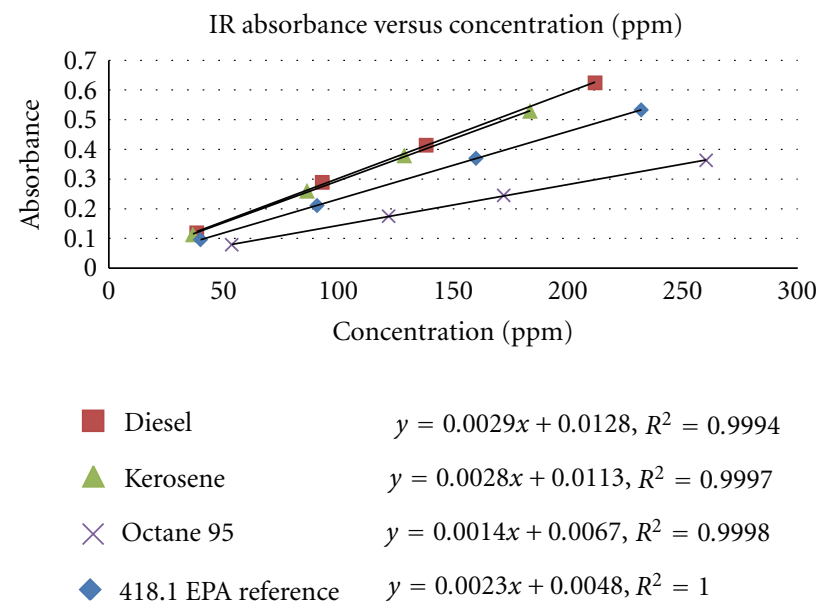

FIGURE 1: IR absorbance versus concentration (ppm).

2.3. IR Absorbance of Diesel, Kerosene, Octane 95, and 418.1 EPA Reference. PHC efficiency to absorb IR radiation depends on the PHC molecules structure. It was important to map these absorptions differences for the contaminants used in this study, relative to the 418.1 EPA reference that is usually used for TPH determination. Diesel, kerosene, octane 95, and the 418.1 EPA reference were mixed with Freon 113 at four different concentration levels each: $\sim 50, \sim 100, \sim 150$, and $\sim 200 \mathrm{ppm}$. Each sample was then measured for its absorbance by a buck scientific 404 analyzer; the results are shown in Figure 1. Since the relation between the absorption and the concentration for each PHC is perfectly linear, (see Figure 1), the absorption was calculated for each PHC for the following concentrations: 50, 100, 150, 200, 250, 300, 350, 400, 450, 500 ppm. Each PHC was then plotted versus the 418.1 EPA reference as shown in Figure 2.

2.4. Conversion of Specific PHC to TPH. Due to the fact that laboratories give results in TPH which is a gross parameter based on the EPA standard that represents a mixture of several PHC, and our soil samples were contaminated by a specific PHC, we need to apply a conversion factor from the specific PHC to the relative gross parameter TPH as seen is Figure 2. This resulted "Projected TPH" value should represent the contamination level of the contaminated samples if the laboratory process was flawless, thus eliminating one major bias factor, which is the difference between IR absorbance efficiency of the 418.1 EPA standard, relative to the specific PHC we used to contaminate the soil as described in the previous section. The conversion equations to project the specific PHC to TPH values in this study (Figure 2) are:

(1) $\mathrm{TPH}(\mathrm{ppm})=$ Diesel $(\mathrm{ppm}) * 1.2609+0.0067$,

(2) $\mathrm{TPH}(\mathrm{ppm})=$ Kerosene $(\mathrm{ppm}) * 1.2174+0.0055$,

(3) $\mathrm{TPH}(\mathrm{ppm})=$ Octane $95 \%(\mathrm{ppm}) * 0.6087+0.0039$,

The calculated projected TPH values are shown in Table 2, and are used for the rest of this study instead of the original specific PHC levels. 


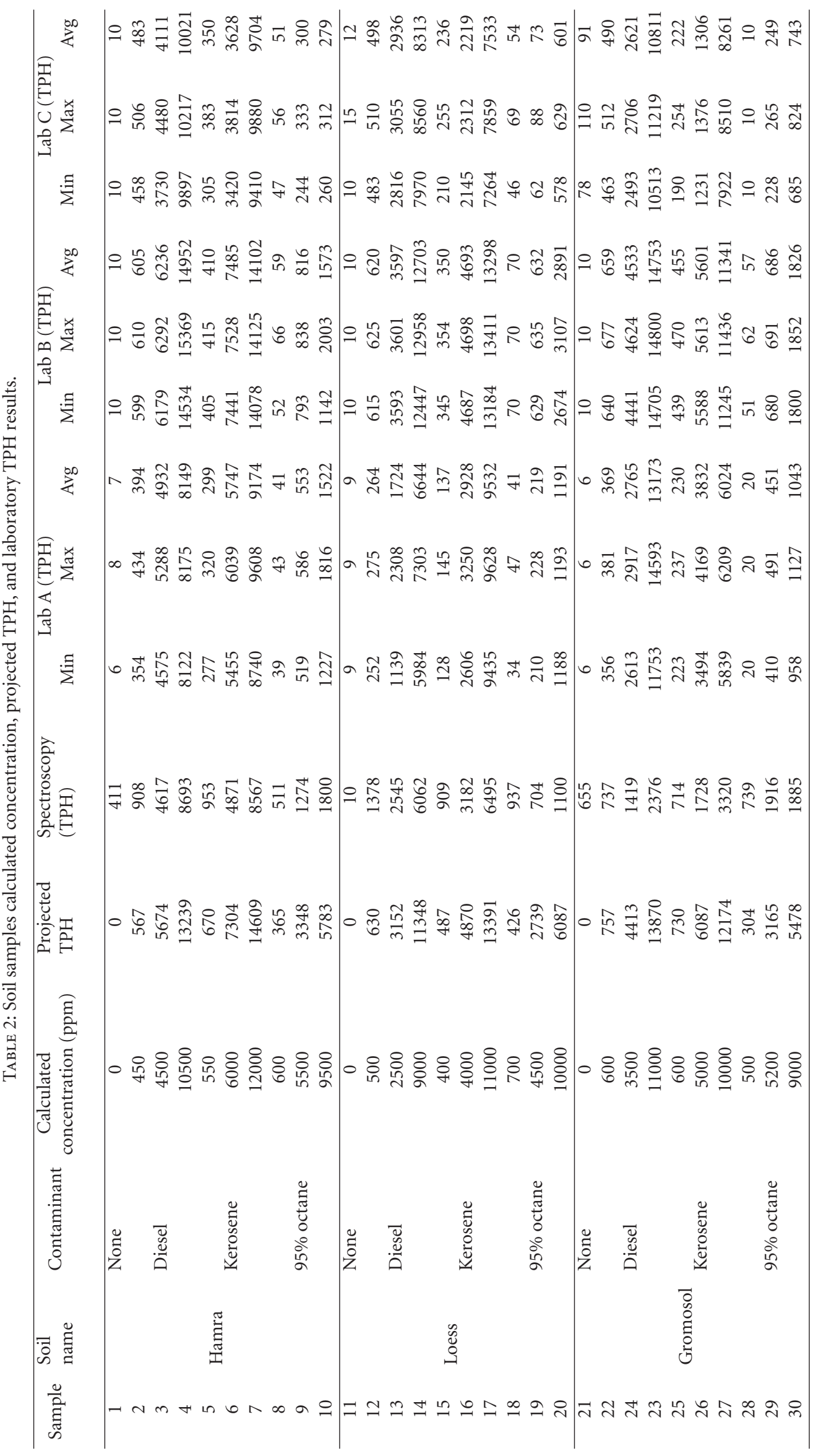




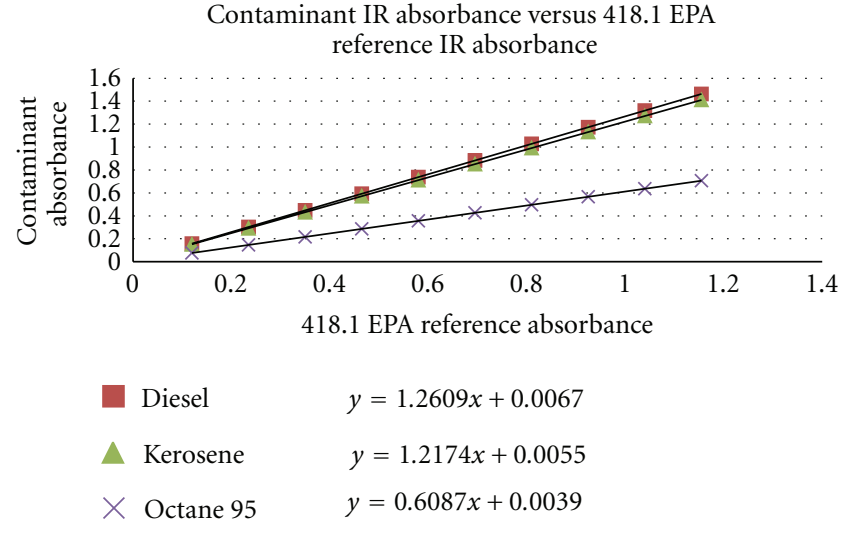

FIgURE 2: Contaminant IR absorbance versus 418.1 EPA reference IR absorbance.

2.5. Intralaboratory Consistency Factors. The contaminated soil samples from each laboratory separately were divided into three groups: low, medium, and high, by the known concentration level, regardless of soil type or contaminant. The intralaboratory consistency was evaluated by four factors.

(1) Average delta: the difference between maximum TPH value and minimum TPH value of each sample in that group, followed by averaging the results of all the samples in that group.

(2) Average deviation: the difference between maximum TPH value and minimum TPH value of each sample in that group, then divided by the average TPH value for that sample, thus normalizing the results. Finally the normalized results of all samples were averaged for all samples in each group.

(3) Maximum delta: same as average delta, but instead of averaging the results for each group, only the maximum value was selected, portraying the "worst case scenario."

(4) Maximum deviation: same as average deviation, but instead of averaging the results for each group, only the maximum value was selected, portraying the "worst case scenario."

Results are shown in Table 3.

2.6. Interlaboratory Consistency Factors. The interlaboratory consistency factors were calculated in the same way the intrafactors were calculated, but instead of taking the samples from each laboratory separately, all samples from all laboratories were joined together, as if they came from the same laboratory. The same four factors: average delta, average deviation, maximum delta, and maximum deviation were calculated as described in the intralaboratory consistency factors section. Results are summarized in Table 4.

2.7. Spectroscopy TPH Measurements. The contaminated soil samples were measured according to TAU's protocol [29] by an ASD Fieldspec pro instrument with an ASD contact probe 3 times, each consisting of 30 measurements that have been averaged; the 3 resulting spectra for each sample were averaged. The average spectrum for each sample was used to predict the TPH level by a PLS model based on several soil types and PHC types, predeveloped in the last few years by the authors. The modeling procedure included five types of soils, three types of PHCs at 50 concentration levels, yielding 750 laboratory prepared samples. An "all possibilities" approach was used for generating robust NIRS models. This approach includes the evaluation of many preprocessing techniques (SNV, MSC, smoothing, absorbance, first and second derivatives, and continuum removal), as well as PLS and ANN modeling methods (i.e., [7, 22, 30-33]).

2.8. General Accuracy. In order to evaluate the reliability of the reflectance spectroscopy method as compared to the common EPA 418.1 method as an environmental monitoring tool, the general accuracy of both methods had to be examined. General accuracy is an important parameter as it determines not only the intra- and interperformances of the laboratories but also portrays the ability of the laboratory to determine the actual contaminant concentration in the sample. General accuracy of TPH measurements done by both reflectance spectroscopy and analytical laboratories, was measured by the same previously mentioned factors used for inter and intra groups as shown in Table 5 (average delta, average deviation, maximum delta, and maximum deviation). The average delta was calculated for each group; by first calculating the delta for each sample in that group (average TPH value-projected TPH value) followed by averaging the results of all the samples in that group. The average deviation was calculated for each group by first calculating the delta for each sample in that group (average TPH value-projected TPH value), then dividing the result with the projected TPH value for that sample, thus normalizing the results. Finally the normalized results of all samples were averaged for each group. The maximum delta and maximum deviation were calculated in the same manner, but instead of averaging the results for each group, only the maximum value was selected portraying the "worst case scenario."

\section{Results and Discussion}

Inner laboratory consistency seems very acceptable with results of under $20 \%$ average deviation for all 3 labs with lab B having the best consistency of under 10\% deviation (Table 3). Although the average deviation is low for all laboratories, in some cases high deviation can occur, even up to $68 \%$ as can be seen in Table 3 (medium concentration samples, Lab A). The interlaboratory consistency on the other hand is far from satisfactory. Average interlaboratory deviation is between $83 \%$ and $103 \%$ and can even reach values of $\sim 200 \%$ in some cases, that is: a Hamra sample contaminated with diesel (Sample 4, Table 2) yielded an average value of 8149 TPH from Lab A and 14952 TPH from Lab B. Both intra and interlaboratory average deviation are presented in Figure 3, performance of Lab A and Lab C are about similar, with better performances by Lab B. General accuracy was also 


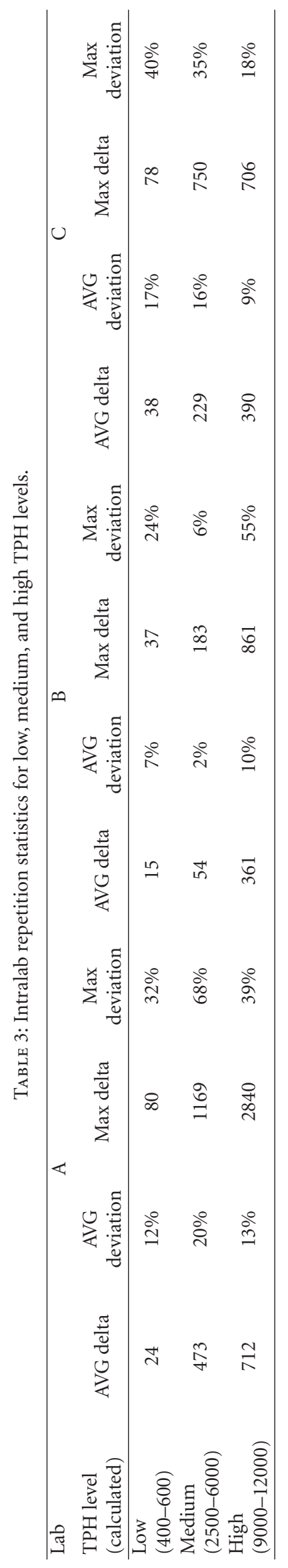


TABLE 4: Interlab repetition statistics for low, medium, and high TPH levels.

\begin{tabular}{lcccc}
\hline TPH level (calculated) & AVG delta & AVG deviation & Max delta & Max deviation \\
\hline Low (400-600) & 190 & $83 \%$ & 373 & $199 \%$ \\
Medium (2500-6000) & 2203 & $103 \%$ & 4382 & $209 \%$ \\
High (9000-12000) & 4564 & $90 \%$ & 7247 & $178 \%$ \\
\hline
\end{tabular}

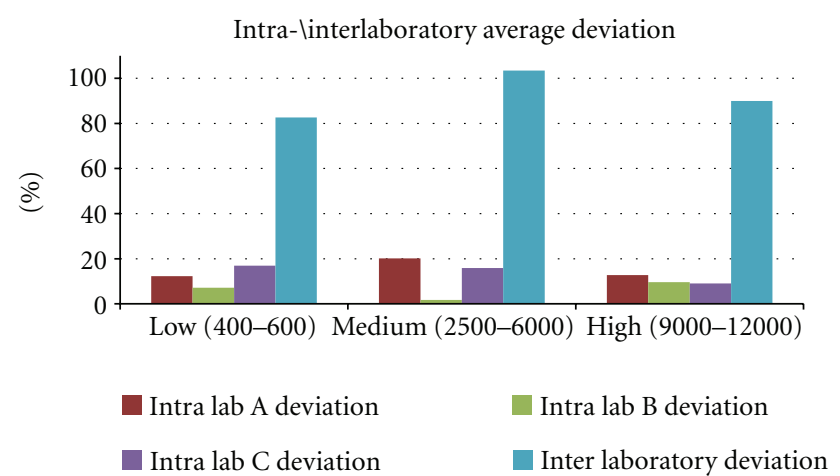

FIGURE 3: Intra-/interlaboratory deviation.

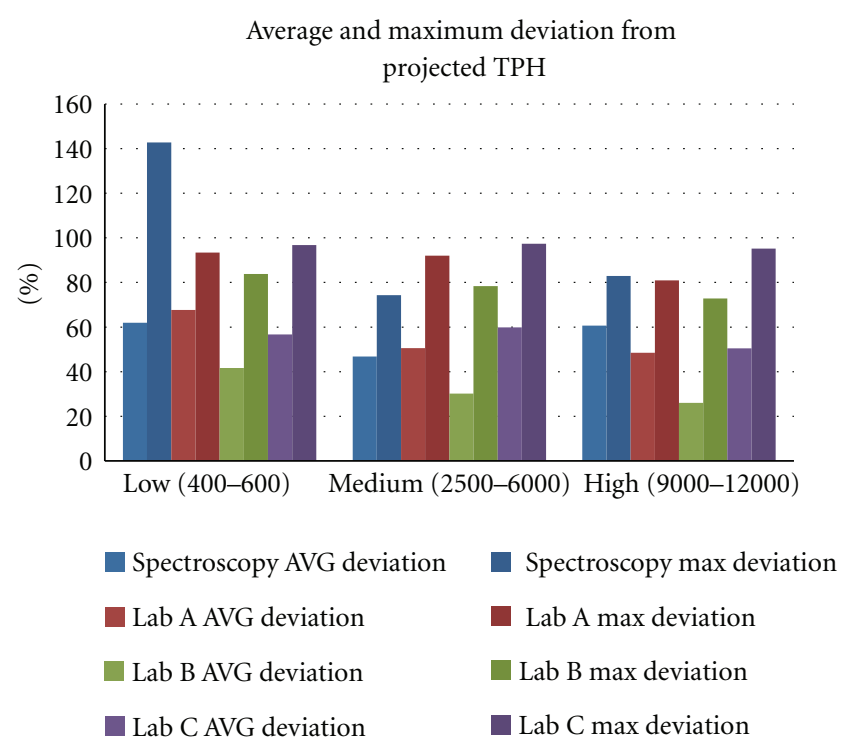

FIgURE 4: Average and maximum deviation from projected TPH.

not satisfactory as seen in Table 5 , average deviation ranged from $26 \%$ up to $68 \%$. Many of the accuracy errors are in measuring $95 \%$ octane fuel; this could be a result of loosing most of the contaminant during the extraction process due to the high volatility nature of this PHC. Performance of all laboratories, including the reflectance spectroscopy method, are almost identical as shown in Figure 4, with Lab B being the most accurate laboratory. Although accuracy was not satisfactory, a good correlation appears when plotting the reflectance spectroscopy and laboratories TPH results against the projected TPH results as demonstrated in Figures 5, 6, and 7. This shows that both the spectroscopy and the laboratories TPH results are consistent and are good predictors of

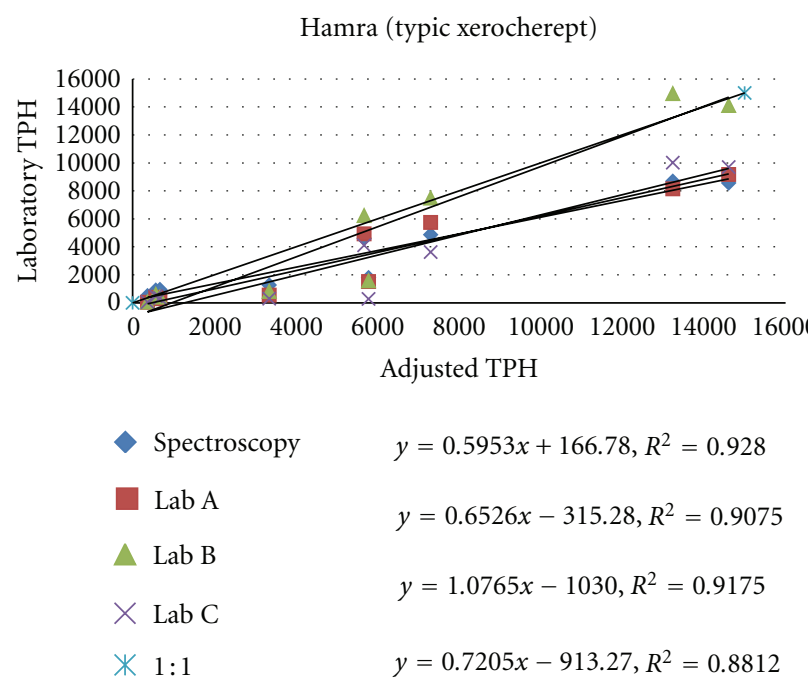

FIgURE 5: Hamra with all PHC types.

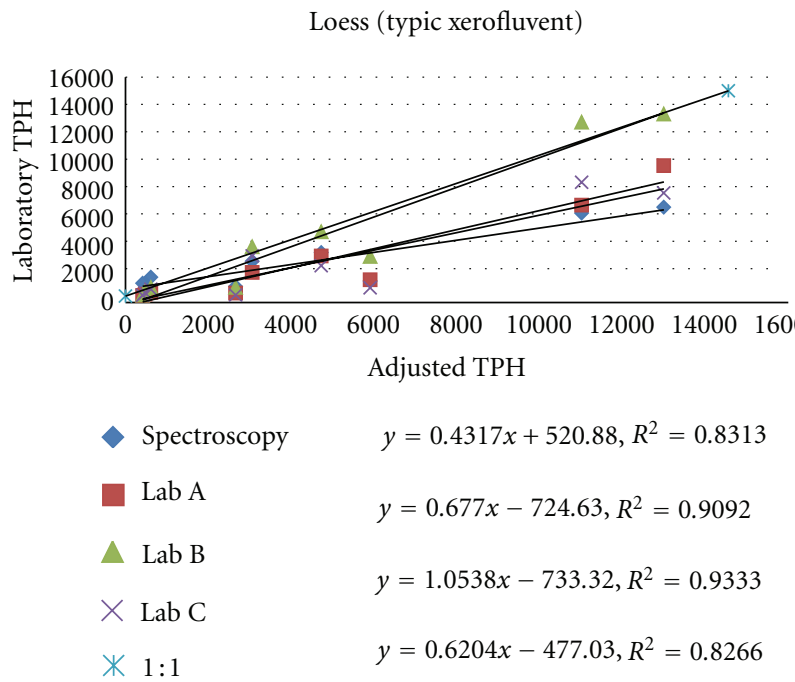

FIgURE 6: Loess with all PHC types.

the contamination levels. Because it is clear that $95 \%$ octane fuel is a problematic contaminant due to its high volatility, when we examine the results while ignoring the $95 \%$ octane contaminated samples, almost perfect correlation coefficient appear (Figures 8, 9, and 10). These correlations between the reflectance spectroscopy and the laboratories TPH results shows consistency of Lab B being always over estimating the projected TPH values, and the reflectance spectroscopy, $\mathrm{Lab} \mathrm{A}$ and Lab $\mathrm{C}$ always under estimating the projected 


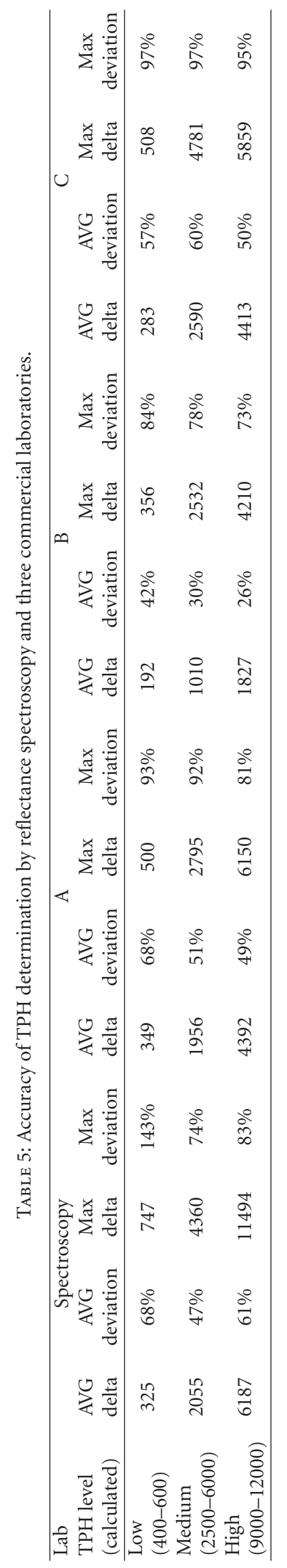



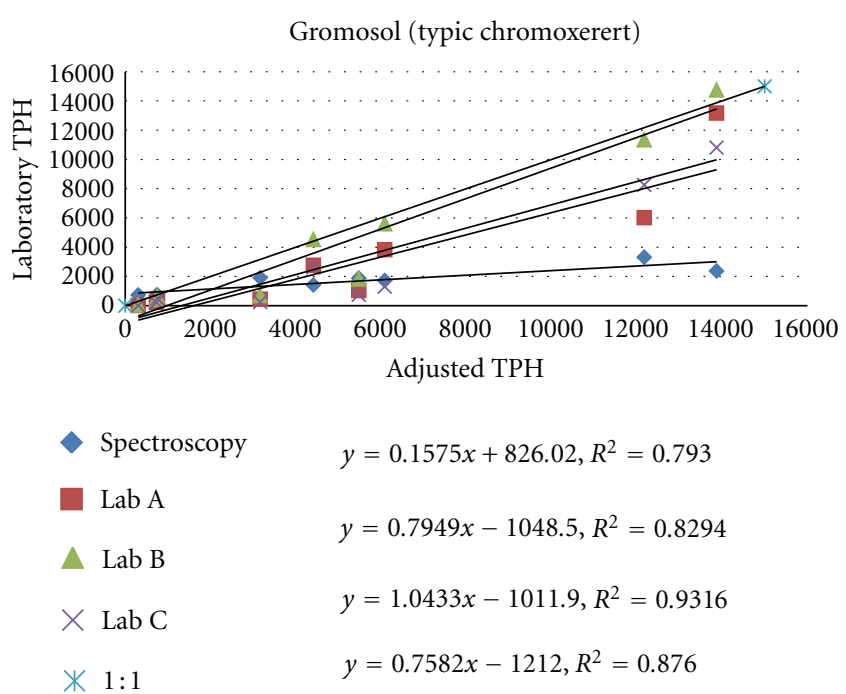

Figure 7: Gromosol with all PHC types.

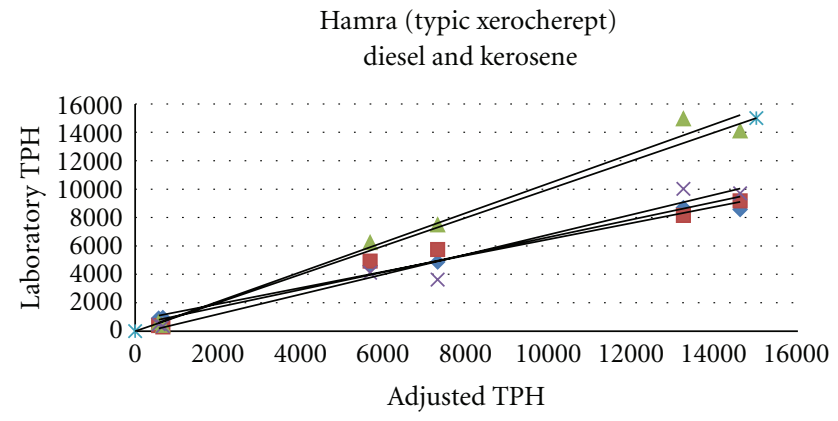

$$
\begin{aligned}
& \text { Spectroscopy } \quad y=0.5696 x+774.64, R^{2}=0.9852 \\
& \text { Lab A } \\
& \triangle \mathrm{Lab} B \\
& \times \text { Lab C } \\
& \text { * } 1: 1 \\
& y=0.6169 x+457.14, R^{2}=0.9665 \\
& y=1.0417 x-5.3423, R^{2}=0.9859 \\
& y=0.7017 x-203.17, R^{2}=0.9687
\end{aligned}
$$

Figure 8: Hamra with diesel and kerosene.

TPH values at almost the same level. As this phenomena being so consistent, it can be corrected by the correlation factors specific for each Laboratory. The result of this study confirms the hypothesis of large variations between laboratories and methods, even though they are properly certified by the authorities. It is interesting to note that with a precise approach, it is possible to account for these variations, correct and calibrate the results to represent the contamination levels accurately, thus enabling reliable comparable results. Reflectance spectroscopy was found to be as good as the traditional method employed by the commercial certified laboratories. Reflectance spectroscopy is a nondestructive method that can be used for rapid, simple, and cost effective TPH determination both in the laboratory and in the field. Moreover, the resent advances in imaging spectroscopy field could enable the adding of a

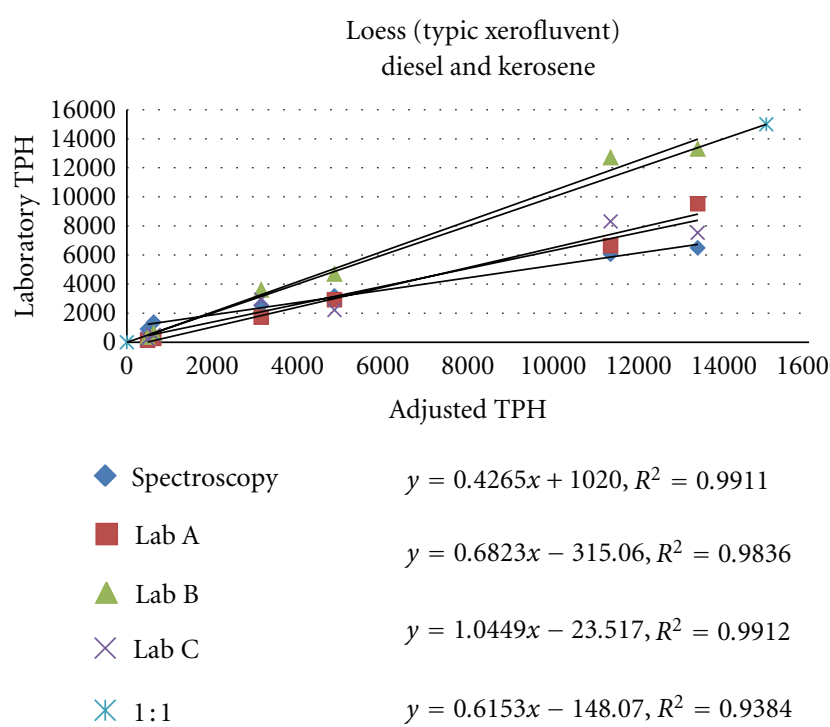

FIGURE 9: Loess with diesel and kerosene.

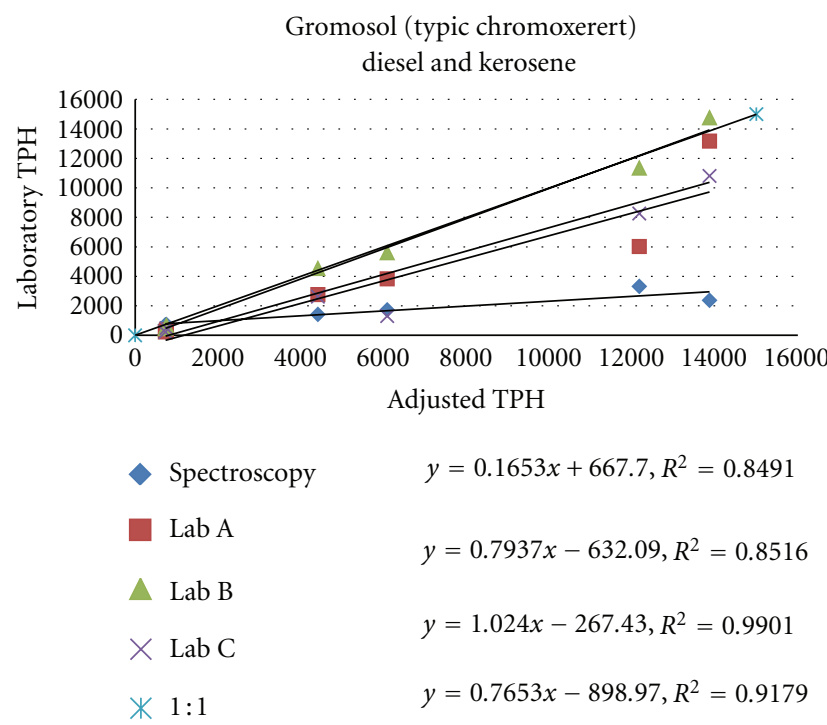

Figure 10: Gromosol with diesel and kerosene.

new spatial dimension for site investigation, opening new frontiers in monitoring PHC contamination in soil.

\section{Conclusion}

While accuracy level is affected by various elements such as laboratory protocols, equipment and personnel, results remain very consistent and can be corrected when certain factors specific for each laboratory are employed. When a new batch of samples needs to be evaluated, a sample of clean soil similar to the same batch, contaminated with the 418.1 EPA standard at two levels can be added to the batch, thus helping to model the bias for this batch and to calibrate the results. Due to the problematic nature of measuring the 95\% octane TPH levels, a PID (Photo Ionization Detector) instrument should be used to accompany each sample to 
help measure the volatile PHC. Reflectance Spectroscopy performed very well in this study (almost the same as Lab A and Lab C), and should be considered as a tool for field screening due to its very low cost per sample, easy operation, ability to work in field conditions, and the possibility of fast measurements and instant results. Reflectance spectroscopy is a nondestructive environmental friendly method; that when coupled with a PID device (for volatile PHC detection) could be used as an excellent screening tool in the field. When using reflectance spectroscopy coupled with PID, contaminated samples should not elude detection. In general the 418.1 EPA method alone should not be used to grant a "clean bill of health" to any contaminated site, but only as a screening and decision-making tool before more expensive methods are employed. It is strongly recommended that any certified laboratory and method will be improved by using a standard protocol suggested in this study, for calibrating the laboratory results to the real contamination level of the soil. Applying these protocols will assure both intra- and interaccurate, consistent, and comparable results.

\section{References}

[1] M. S. Hutcheson, D. Pedersen, N. D. Anastas, J. Fitzgerald, and D. Silverman, "Beyond TPH: health-based evaluation of petroleum hydrocarbon exposures," Regulatory Toxicology and Pharmacology, vol. 24, no. 1, pp. 85-101, 1996.

[2] P. Boffetta, N. Jourenkova, and P. Gustavsson, "Cancer risk from occupational and environmental exposure to polycyclic aromatic hydrocarbons," Cancer Causes and Control, vol. 8, no. 3, pp. 444-472, 1997.

[3] G. D. Ritchie, K. R. Still, W. K. Alexander et al., "A review of the neurotoxicity risk of selected hydrocarbon fuels," Journal of Toxicology and Environmental Health B, vol. 4, no. 3, pp. 223312, 2001.

[4] Environmental Sciences Division, Use of Gross Parameters for Assessment of Hydrocarbon Contamination of Soils in Alberta, Oxford, UK, 1993.

[5] United States Environmental Protection Agency (USEPA), Test Method for Evaluating Total Recoverable Petroleum Hydrocarbon, Method 418.1 (Spectrophotometric, Infrared), Government Printing Office, Washington, DC, USA, 1978.

[6] United States Environmental Protection Agency (USEPA), Methods for Chemical Analysis of Water and Wastes, Government Printing Office, Washington, DC, USA, 1983.

[7] G. Schwartz, G. Eshel, and E. Ben-Dor, "Reflectance spectroscopy as a tool for monitoring contaminated soils," in Soil Contamination, Intech, 2011.

[8] R. S. G. Gómez, T. Pandiyan, V. E. A. Iris, V. Luna-Pabello, and C. D. de Bazúa, "Spectroscopic determination of polyaromatic compounds in petroleum contaminated soils," Water, Air, and Soil Pollution, vol. 158, no. 1, pp. 137-151, 2004.

[9] J. Krupcík, P. Oswald, D. Oktavec, and D. W. Armstrong, "Calibration of GC-FID and IR spectrometric methods for determination of high boiling petroleum hydrocarbons in environmental samples," Water, Air, and Soil Pollution, vol. 153, no. 1-4, pp. 329-341, 2004.

[10] G. Xie, M. J. Barcelona, and J. Fang, "Quantification and interpretation of total petroleum hydrocarbons in sediment samples by a GC/MS method and comparison with EPA 418.1 and a rapid field method," Analytical Chemistry, vol. 71, no. 9, pp. 1899-1904, 1999.

[11] P. Lambert, M. Fingas, and M. Goldthorp, "An evaluation of field total petroleum hydrocarbon (TPH) systems," Journal of Hazardous Materials, vol. 83, no. 1-2, pp. 65-81, 2001.

[12] E. Saari, P. Perämäki, and J. Jalonen, "A comparative study of solvent extraction of total petroleum hydrocarbons in soil," Microchimica Acta, vol. 158, no. 3-4, pp. 261-268, 2007.

[13] M. Villalobos, A. P. Avila-Forcada, and M. E. Gutierrez-Ruiz, "An improved gravimetric method to determine total petroleum hydrocarbons in contaminated soils," Water, Air, and Soil Pollution, vol. 194, no. 1-4, pp. 151-161, 2008.

[14] E. A. Cloutis, "Spectral reflectance properties of hydrocarbons: remote-sensing implications," Science, vol. 245, no. 4914, pp. 165-168, 1989.

[15] I. Schneider, G. Nau, T. V. V. King, and I. Aggarwal, "Fiberoptic near-infrared reflectance sensor for detection of organics in soils," IEEE Photonics Technology Letters, vol. 7, no. 1, pp. 87-89, 1995.

[16] B. R. Stallard, M. J. Garcia, and S. Kaushik, "Near-IR reflectance spectroscopy for the determination of motor oil contamination in sandy loam," Applied Spectroscopy, vol. 50, no. 3, pp. 334-338, 1996.

[17] Z. Zwanziger and F. Heidrun, "Near infrared spectroscopy of fuel contaminated sand and soil. I. Preliminary results and calibration study," Journal of Near Infrared Spectroscopy, vol. 6, no. 1-4, pp. 189-197, 1998.

[18] D. F. Malley, K. N. Hunter, and G. R. B. Webster, "Analysis of diesel fuel contamination in soils by near-infrared reflectance spectrometry and solid phase microextraction-gas chromatography," Soil and Sediment Contamination, vol. 8, no. 4, pp. 481-489, 1999.

[19] B. Hörig, F. Kühn, F. Oschütz, and F. Lehmann, "HyMap hyperspectral remote sensing to detect hydrocarbons," International Journal of Remote Sensing, vol. 22, no. 8, pp. 14131422, 2001.

[20] F. Kühn, K. Oppermann, and B. Hörig, "Hydrocarbon index-an algorithm for hyperspectral detection of hydrocarbons," International Journal of Remote Sensing, vol. 25, no. 12, pp. 2467-2473, 2004.

[21] K. H. Winkelmann, On the applicability of imaging spectrometry for the detection and investigation of contaminated sites with particular consideration given to the detection of fuel hydrocarbon contaminants in soil, $\mathrm{Ph} . \mathrm{D}$. thesis, Brandenburgische Technische Universität Cottbus, 2005.

[22] G. Schwartz, G. Eshel, M. Ben-Haim, and E. Ben-Dor, "Rapid methods for classification and quantitative assessment of petroleum hydrocarbons pollution in soil samples using reflectance spectroscopy," EGU 2009-11441-2, Vienna, Austria, 2009.

[23] S. Chakraborty, D. C. Weindorf, C. L. S. Morgan et al., "Rapid identification of oil-contaminated soils using visible nearinfrared diffuse reflectance spectroscopy," Journal of Environmental Quality, vol. 39, no. 4, pp. 1378-1387, 2010.

[24] T. Lammoglia and C. R. de S. Filho, "Spectroscopic characterization of oils yielded from Brazilian offshore basins: potential applications of remote sensing," Remote Sensing of Environment, vol. 115, no. 10, pp. 2525-2535, 2011.

[25] J. Dan and H. Koyumdjisky, "The soils of israel and their distribution," European Journal of Soil Science, vol. 14, no. 1, pp. 12-20, 1963.

[26] S. S. Staff, Keys to Soil Taxonomy, Government Printing Office, 2010. 
[27] D. L. Carter, M. M. Mortland, and W. D. Kemper, "Specific surface," in Methods of Soil Analysis Part I. Soil Science, A. Klute, Ed., pp. 413-422, Society of America, Madison, Wis, USA, 1986.

[28] G. Eshel, G. J. Levy, U. Mingelgrin, and M. J. Singer, "Critical evaluation of the use of laser diffraction for particle-size distribution analysis," Soil Science Society of America Journal, vol. 68, no. 3, pp. 736-743, 2004.

[29] A. Pimstein, E. Ben-Dor, and G. Notesco, "Performance of three identical spectrometers in retrieving soil reflectance under laboratory conditions," Soil Science Society of America Journal, vol. 75, no. 2, pp. 746-759, 2011.

[30] G. Schwartz, G. Eshel, M. Ben-Haim, and E. Ben-Dor, Reflectance Spectroscopy as a Rapid Tool for Qualitative Mapping and Classification of Hydrocarbons Soil Contamination, Tel Aviv, Israel, 2009.

[31] G. Schwartz, G. Eshel, M. Ben-Haim, and E. Ben-Dor, Quantitative Assessment of Petroleum Hydrocarbons in Situ by Diffused Reflectance Spectroscopy and a Penetrating Optical Sensor, GFZ, Potsdam, Germany, 2010.

[32] G. Schwartz, G. Eshel, and E. Ben-Dor, An Operational Spectral Based Model to Predict Soil Petroleum Hydrocarbon Content in Field Samples, Edinburgh, Scotland, 2011.

[33] G. Schwartz, Reflectance spectroscopy as a rapid tool for qualitative mapping and classification of hydrocarbons soil contamination, Ph.D. thesis, Tel Aviv University, 2012. 

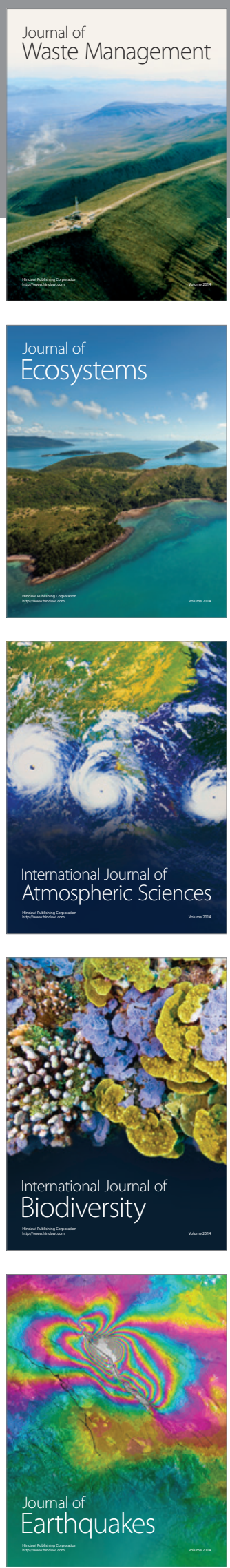
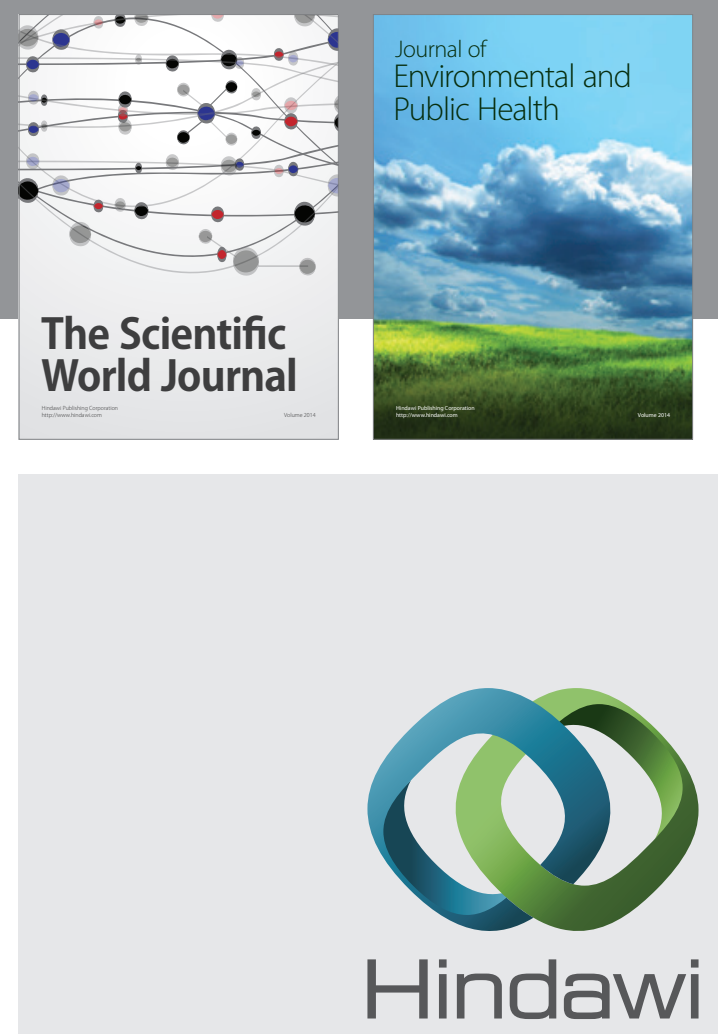

Submit your manuscripts at

http://www.hindawi.com
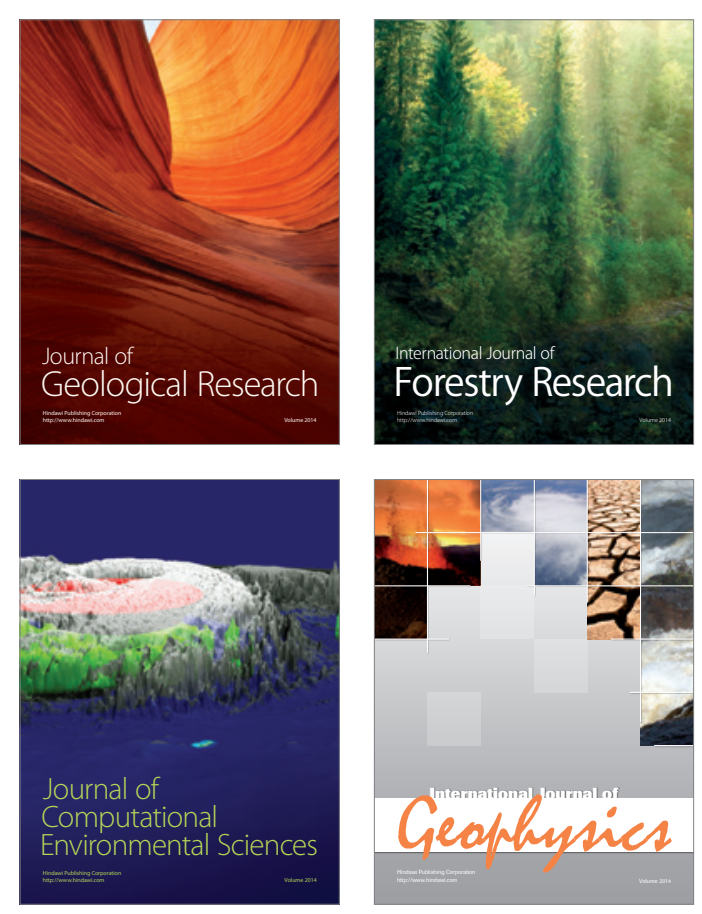
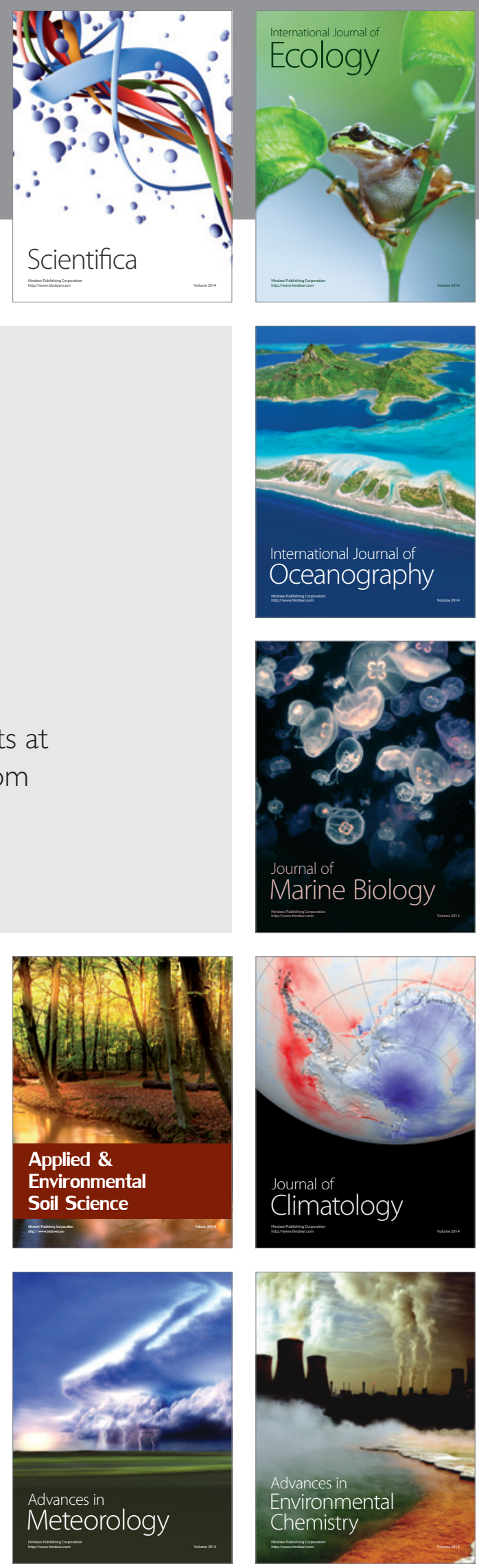